\title{
ORIGINAL RESEARCH \\ Variations in the Course of the Cervical Vagus Nerve on Thyroid Ultrasonography
}

J.K. Park

S.Y. Jeong

J.-H. Lee

G.C. Lim

J.W. Chang
BACKGROUND AND PURPOSE: Only 1 ultrasonography study that described the variation of the VN had been published at the time our research was begun. The purpose of this study was to evaluate the incidence and type of variation in the course of the cervical VN on thyroid ultrasonography.

MATERIALS AND METHODS: From August 2009 to September 2010, 163 consecutive patients were evaluated by sonography for the screening and characterization of thyroid nodules (mean age, $49.0 \pm$ 14.4 years, male:female, 20:143). Two types of variation were defined as follows: 1) anterior variation, when the course of the VNs changed from the typical location to an anterior location in front of the CCA; and 2) medial variation, when the course of the VNs changed from the typical location to a location medial to the CCA (between CCA and thyroid gland). The incidence of the each variation was studied.

RESULTS: Variation in the course of the VN occurred in 5.5\% (18/326) of cases. The anterior variation was observed in $4.3 \%(14 / 326$, right:left $=4: 10)$, and the medial variation was observed in $1.2 \%$ (4/326, right:left $=3: 1)$. For both variations, the VN was close to or nearly abutted the thyroid gland after it changed course.

CONCLUSIONS: Variation in the course of the cervical VN could be assessed by ultrasonography. Two variations were observed in $5.5 \%$ of cases. The anterior variation was more common than the medial variation.

ABBREVIATIONS: CCA = common carotid artery; IJV = internal jugular vein; RFA = radiofrequency ablation; $\mathrm{VN}=$ vagus nerve
$T^{1}$ he VN descends within the carotid sheath in the neck. From the jugular foramen, it has a relatively straight course up to the thorax. In the carotid sheath, it lies posteriorly in the groove between the carotid artery (internal, common) and the IJV. Although the diameter of the VN is approximately $2 \mathrm{~mm}$, the normal $\mathrm{VN}$ usually can be imaged by only sonography. ${ }^{1,2}$ On longitudinal scan, the VN appears as a striated structure, due to the hypoechoic parallel nervous fascicles separated by a hyperechoic perineurium. On transverse scans, the VN has a honeycomb appearance with 2 to 4 hypoechoic rounded fascicles surrounded by a hyperechoic epineurium. ${ }^{2}$

Injury of the $\mathrm{VN}$ and its branches can occasionally occur during carotid endarterectomy. ${ }^{3,4}$ In addition, the $\mathrm{VN}$ is relatively close to the surgical field of a thyroidectomy. Therefore, knowledge of the anatomic variation in the course of the $\mathrm{VN}$ may be important to reduce the risk of $\mathrm{VN}$ injury. Variation in the course of the VN is very rare, and only 1 variation, an anterior location of the $\mathrm{VN}$ in front of the CCA, has been reported previously. ${ }^{2,5-7}$ The purpose of this study was to evaluate the incidence and type of variation in the course of the cervical VN on thyroid ultrasonography.

Received August 13, 2010; accepted November 11

From the Departments of Radiology (J.K.P., S.Y.J.), Otolaryngology-Head and Neck Surgery (G.C.L.), and Thoracic Surgery (J.W.C.), Jeju National University College of Medicine, Jeju, Korea; and Department of Radiology (J.-H.L.), Asan Medical Center, University of Ulsan College of Medicine, Seoul, Korea.

Please address correspondence to Ji Kang Park, Department of Radiology, Jeju National University Hospital, Jeju National University School of Medicine, 1753-3, Ara-1-dong, Jeju-Si, Jeju Special Self-Governing Province, 690-716, Korea; e-mail: jkcontrast@ naver.com

Indicates article with supplemental on-line figure.

DOI 10.3174/ajnr.A2476

\section{Materials and Methods}

This study was approved by the institutional review board. From August 2009 to September 2010, 163 consecutive patients were evaluated by sonography for the screening and characterization of thyroid nodules (mean age, $49.0 \pm 14.4$ years, male:female, 20 : 143). Acuson S2000 (Siemens Medical Solutions, Mountain View, California) and ATL HDI 5000 (Philips, Bothell, Washington) were used for the evaluation. Two radiologists assessed the VN by sonography and identified the VN by the typical sonographic features and location according to previous literature ${ }^{2}$ during sonography examination. We defined 2 types of variation as follows: 1 ) anterior variation, when the course of the $\mathrm{VN}$ changed from the typical location to an anterior location in front of the CCA during its descending course (Fig 1); and 2) medial variation, when the course of the VN changed from the typical location to a medial location relative to the CCA (between CCA and thyroid gland) during its descending course (Fig 2). We evaluated the incidence of the each variation of the $\mathrm{VN}$.

\section{Results}

In total, $326 \mathrm{VNs}$ were easily identified on ultrasonography. The variations in the course of the VN were identified in $5.5 \%(18 / 326)$ of cases. Anterior variation was observed in $4.3 \%(14 / 326$, right:left $=4: 10)$ and medial variation in $1.2 \%$ of cases $(4 / 326$, right:left $=3: 1)$. Bilateral variation was not observed. For the anterior variation, the VN went over the CCA when it changed course (Fig 1). For the medial variation, the $\mathrm{VN}$ went across under the CCA when it changed course (Fig 2). For both variations, especially in the medial variation, the $\mathrm{VN}$ was close to or nearly abutted the thyroid gland after it changed course. For both variations, the course of the VN began to change superior to or near to the upper part of the thyroid gland. In one case, 

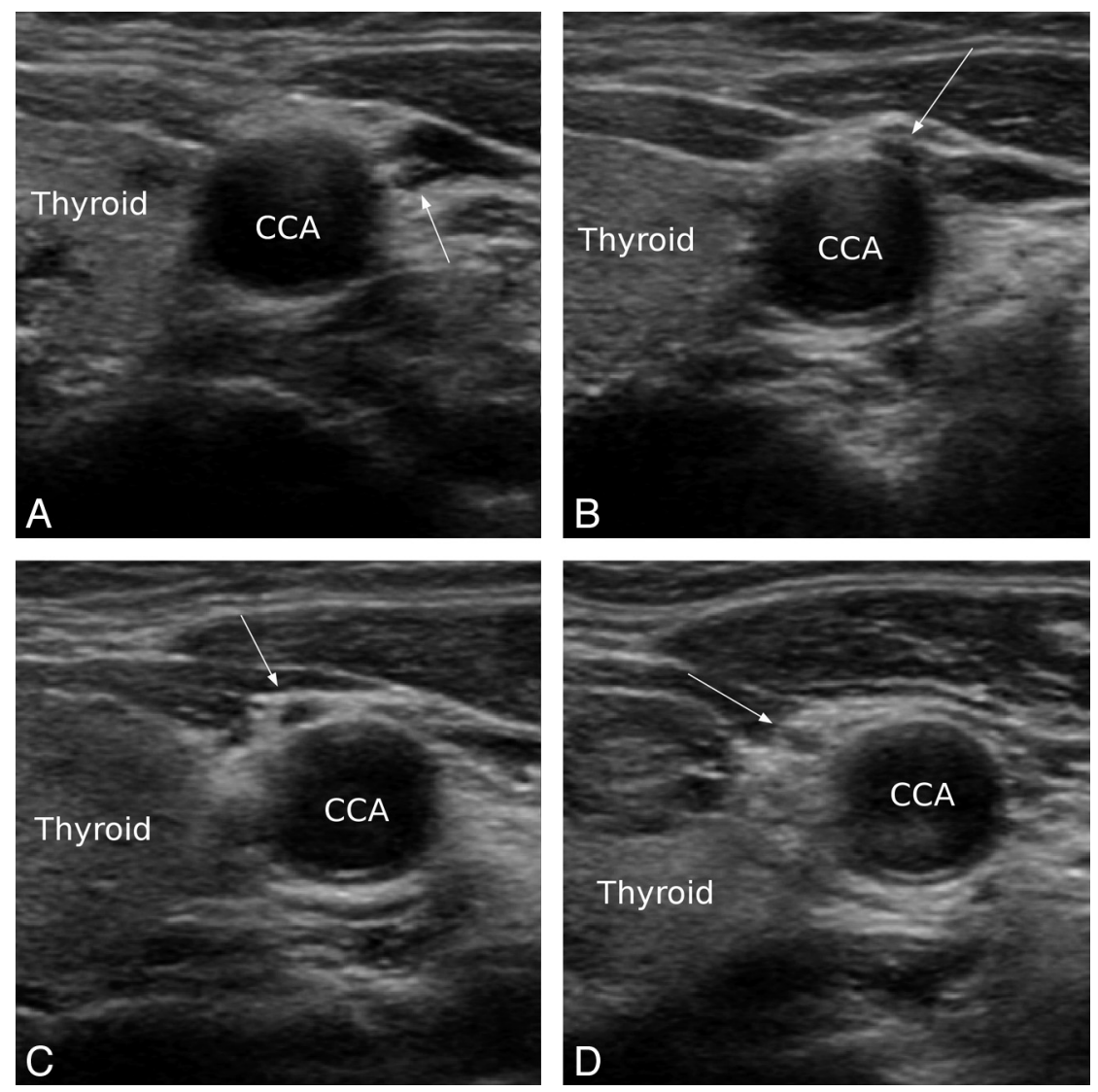

Fig 1. Anterior variation of the VN in transaxial ultrasonography. In the upper pole area of the thyroid $(A)$, VN (arrow) is located between CCA and IJV. Then the VN changes its course, traversing the space between the CCA and IJV, in front of the CCA $(B, C)$. After it changes course, the VN is located anterior and medial to CCA and is close to the thyroid gland $(D)$.

despite the normal VN position, the whole carotid sheath was inwardly rotated, and the VN was very close to the thyroid gland. In this patient, the artery had a tortuous course and the thyroid gland was enlarged (Fig 3).

\section{Discussion}

The results of this study showed that the course of the cervical $\mathrm{VN}$ varied in $5.5 \%$ of cases. This frequency is higher than previously reported $(0.7 \%){ }^{2}$ Anterior variation was more common than medial variation in this study. The anterior variation in this study was identical to the variation reported in the previous sonography study. However, medial variation is a newly identified variation; there is no prior report of this. The previous reports suggested the possibility of a close relationship between the thyroid gland and cervical $\mathrm{VN}$ during embryologic development. ${ }^{78}$ This suggestion was based on an animal study where neuronal cells from the distal vagal ganglion originated from parafollicular $\mathrm{C}$ cells of the thyroid gland. ${ }^{8}$ In the anterior variation, the $\mathrm{VN}$ was close to the thyroid gland after it changed course in a previous sonography study. ${ }^{2}$ In addition, it was shown that the $\mathrm{VN}$ with the anterior variation was loosely connected to the thyroid gland by connective tissue in an autopsy study. ${ }^{7}$ The results of this study are consistent with prior reports. Although there have been no surgical reports confirming a connection between thyroid gland and $\mathrm{VN}$ with variation, there might be an association between thyroid gland and the variation of the VN.
In this study, the $\mathrm{VN}$ was easily identified and traced by ultrasonography. Iatrogenic injury to the recurrent laryngeal nerve branch of the VN has been reported in thyroidectomy, carotid endarterectomy, and anterior cervical spine surgery. The incidence of the recurrent laryngeal nerve branch ranged from $<1 \%$ to $6 \%$ for thyroidectomy, from $<1 \%$ to $8 \%$ for carotid endarterectomy, and from $1.3 \%$ to $2.6 \%$ for anterior cervical spine surgery. ${ }^{4,9-12}$ The recurrent laryngeal fibers are medially placed in the vagal trunk. ${ }^{3,13}$ For anterior variation, the VN was superficial and medial to the CCA, and nerve injury may occur during dissection by a diathermy burn or mechanical stretching during carotid endarterectomy. ${ }^{3,13}$ For medial variation, the $\mathrm{VN}$ was deep and posterior to the CCA, and nerve injury may occur by sharp dissection for mobilization of the CCA during endarterectomy., ${ }^{3,13}$ For thyroid surgery, the $\mathrm{VN}$ is located within the carotid sheath; the surgeons usually pay less attention to the VNs than recurrent laryngeal nerve. However, for both variations, the $\mathrm{VN}$ is near the thyroid gland and may be injured during lateral dissection during a thyroidectomy. Recently, RFA has been used for treatment of thyroid nodules. ${ }^{14,15}$ The tip of a RFA electrode may be placed close to the CCA when the nodule is located in the lateral portion of the thyroid gland. In that case, the risk for injury to the $\mathrm{VN}$ may be considered in cases with variation in the course of the $\mathrm{VN}$ (On-line Fig 1). Therefore, the information about the variant course of the $\mathrm{VN}$ may be important to prevent injury to the $\mathrm{VN}$ in patients undergoing thyroidectomy, ca- 

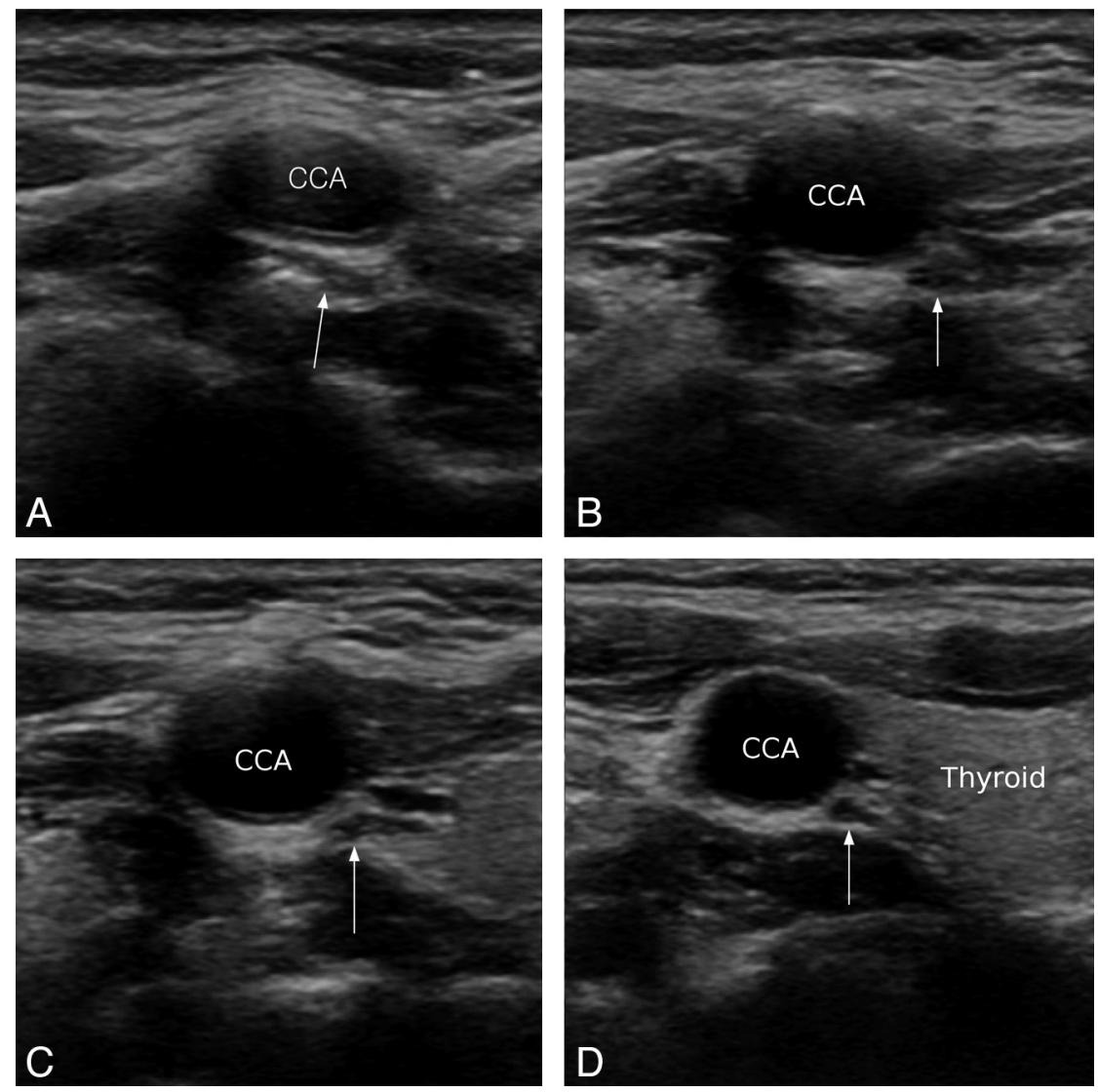

Fig 2. Medial variation of the VN in transaxial ultrasonography. $A$, VN changes its course under the CCA (arrow). After it changes course, the VN is located medial to the CCA and is close to the thyroid gland until it enters the thorax $(B-D)$.
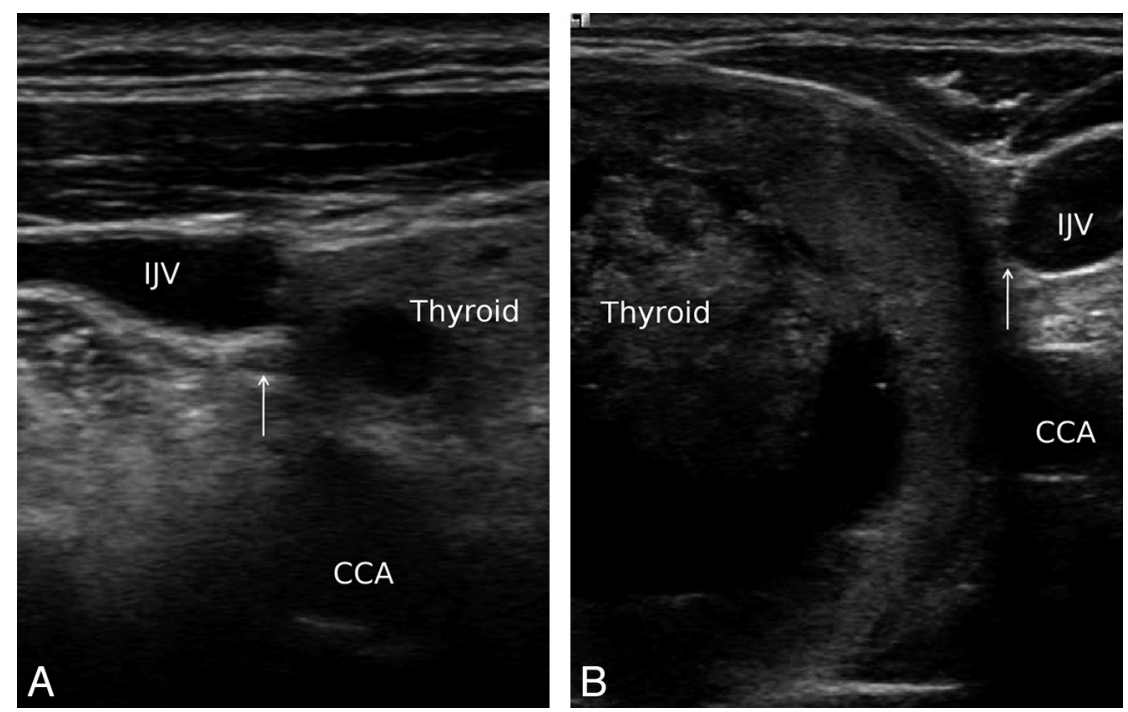

Fig 3. VN (arrow) is located between the IJV and CCA on both sides in transaxial ultrasonography. The whole carotid sheath is inwardly rotated, and the VN is very close to the thyroid gland.

rotid endarterectomy, or thyroid RFA. It may be necessary to evaluate the variation in the course of the VN with sonography before such procedures. In one case in this study, despite a normal position, the $\mathrm{VN}$ was close to the thyroid gland due to the inward rotation of the whole carotid sheath. In this case, the CCA had a tortuous course and was angulated, and the thyroid gland was enlarged due to a goiter. It is possible that the course of the VN could be angulated and displaced by the change of the surrounding environment such as the CCA and the thyroid gland. In that case, the change of the course of the VN can be easily determined by ultrasonography. In this study, there was no correlation between the sonography findings and surgical findings. The incidence of the variation in the course of the $\mathrm{VN}$ was not insignificant; however, there have been no reports on associated complication during carotid endarterectomy or thyroidectomy procedures. Further study 
may be needed to confirm the incidence and the association of such variations with surgical findings and outcomes of carotid endarterectomy and thyroidectomy procedures.

\section{Conclusions}

The variation in the course of cervical VNs can be assessed by ultrasonography. Variation occurred in 5.5\% of our cases. Anterior variation was more common than medial variation. The knowledge of variation in the course of the VN may be helpful for carotid endarterectomy or thyroidectomy in terms of reduction of the risk of injury to the $\mathrm{VN}$.

\section{References}

1. Knappertz VA, Tegeler $\mathrm{CH}$, Hardin SJ, et al. Vagus nerve imaging with ultrasound: anatomic and in vivo validation. Otolaryngol Head Neck Surg 1998;118:82-85

2. Giovagnorio F, Martinoli C. Sonography of the cervical vagus nerve: normal appearance and abnormal findings. AJR Am J Roentgenol 2001;176:745-49

3. AbuRahma AF, Lim RY. Management of vagus nerve injury afer carotid endarterectomy. Surgery 1996;119:245-47

4. Ballotta E, Da Giau G, Renon L, et al. Cranial and cervical nerve injuries after carotid endarterectomy: a prospective study. Surgery 1999;125:85-91
5. Gibson A. Bilateral abnormal relationship of the vagus nerve in its cervical portion. J Anat Physiol 1915;49:389-92

6. Veleanu C, Dinulescu T, Zolog I. Vagus nerve passing in front of the left lobe of the thyroid gland. Anat Anz 1977;141:84-85

7. Tubbs RS, Loukas M, Shoja MM, et al. An unreported variation of the cervical vagus nerve: anatomical and histological observations. Folia Morphol (Warsz) 2007;66:155-57

8. Kameda Y. Evidence to support the distal vagal ganglion as the origin of C cells of the ultimobranchial gland in the chick. J Comp Neurol 1995;359:1-14

9. Miller MC, Spiegel JR. Identification and monitoring of the recurrent laryngeal nerve during thyroidectomy. Surg Oncol Clin N Am 2008;17:121-44, viii-ix

10. Assadian A, Senekowitsch C, Pfaffelmeyer N, et al. Incidence of cranial nerve injuries after carotid eversion endarterectomy with a transverse skin incision under regional anaesthesia. Eur J Vasc Endovasc Surg 2004;28:421-24

11. Kilburg C, Sullivan HG, Mathiason MA. Effect of approach side during anterior cervical discectomy and fusion on the incidence of recurrent laryngeal nerve injury. J Neurosurg Spine 2006;4:273-77

12. Kahraman S, Sirin S, Erdogan E, et al. Is dysphonia permanent or temporary after anterior cervical approach? Eur Spine J 2007;16:2092-95

13. Schauber MD, Fontenelle LJ, Solomon JW, et al. Cranial/cervical nerve dysfunction after carotid endarterectomy. J Vasc Surg 1997;25:481-87

14. Jeong WK, Baek JH, Rhim H, et al. Radiofrequency ablation of benign thyroid nodules: safety and imaging follow-up in 236 patients. Eur Radiol 2008;18:1244-50

15. Lee JH, Kim YS, Lee D, et al. Radiofrequency ablation (RFA) of benign thyroid nodules in patients with incompletely resolved clinical problems after ethanol ablation (EA). World J Surg 34:1488-93 